\title{
Performance analysis of spectral/spatial of OCDMA system using 2D hybrid ZCC/MD code
}

\author{
Rima. Matem, S. A. Aljunid, M. N Junita, C. B.M Rashidi, Israa Shihab Aqrab \\ Advanced Communication Engineering Center of Excellence School of Computer and \\ Communication Engineering (ACE CoE-SCCE), Universiti Malaysia Perlis (UniMAP), Perlis, Malaysia
}

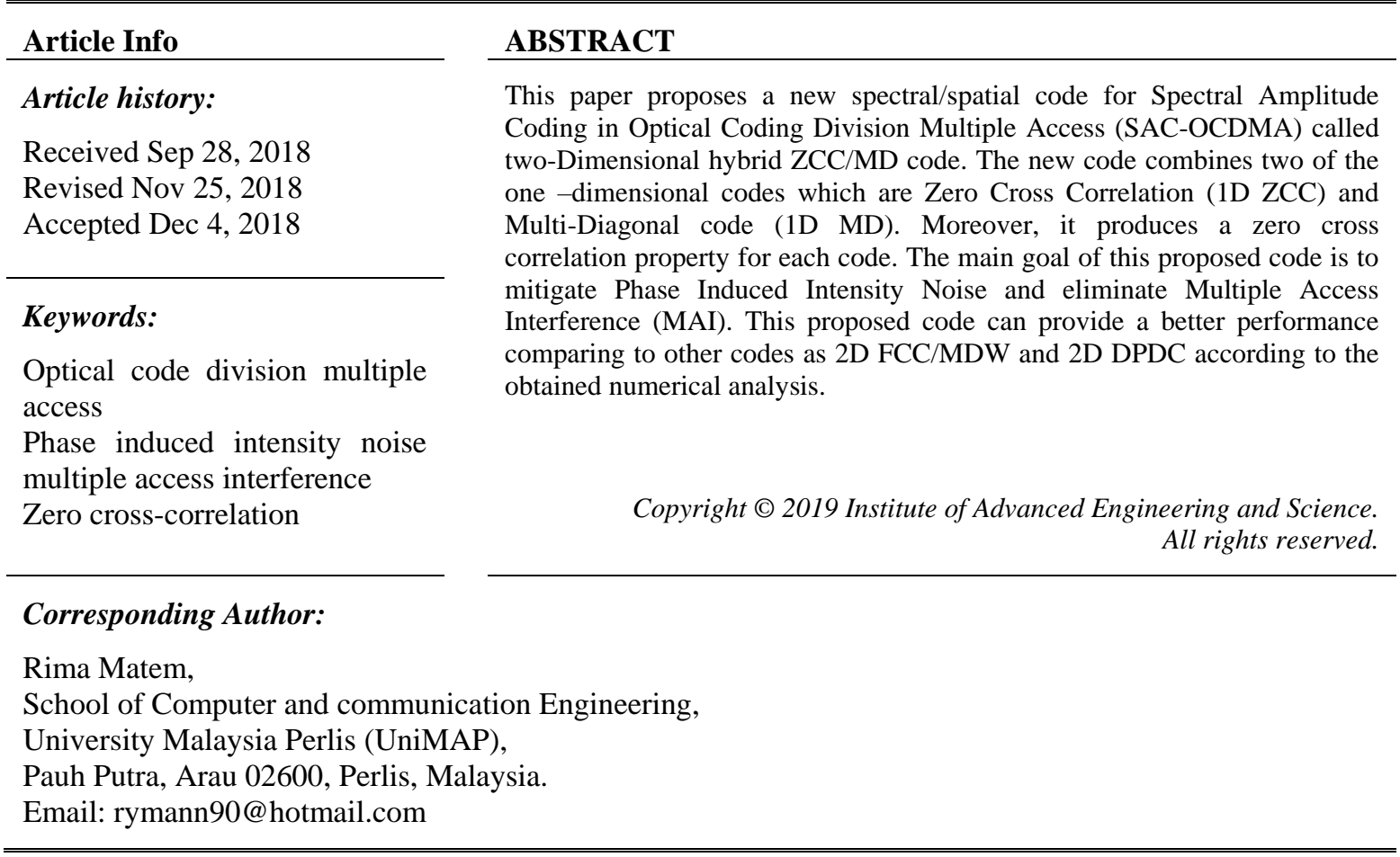

\section{INTRODUCTION}

The key goal in developing Optical Code Division Multiple Access (OCDMA) is to overcome some disadvantages in a communication network through optical fiber such as low-security level when multiple users access the same bandwidth, low rate transmitting speed, and complexity designing. OCDMA with its characteristic offers a lot of advantages as asynchronous and simultaneous access of multiple users to the network, broadband access and low losses, the transmission with high level of security and facilitation of the realization of many services so OCDMA has been introduced to respond to many requirements in optical fiber communication [1]. OCDMA system can be classified on coherent and incoherent based on the property of its respective optical signal [2], many schemes have been proposed where Spectral Amplitude Coding is one of these schemes (SAC-OCDMA) [3, 4]. SAC-OCDMA has been attracted from a huge research according to its features but it can be affected by many factors such as Phase Induced Intensity Noise (PIIN) and Multiple Access Interference (MAI) which are the main challenges of OCDMA system [5, 6]. To achieve this goal two-dimensional codes are developed to overcome deficiencies of one-dimensional codes which are introduced as 2D Diluted Perfect Difference (2D DPD) [7], 2D Modified Double Weight (2D MDW) [8, 9], 2D hybrid code FCC/MDW [10], 2D Dynamic Cyclic Shift code (2D DCS) [11]. In this paper, the proposed code 2D ZCC/MD is developed to mitigate PIIN and suppress MAI to attain high performance. The current paper is arranged as follow, the second section focuses about 2D ZCC/MD construction combining 1D MD [12] and 1D ZCC $[13,14]$. The third section describes results and discussion. Finally, the fourth section the conclusion of this study. 


\section{HYBRID ZCC/MD CODE}

Based on the combination of 1D MD [12] and 1D ZCC [13, 14] 2D ZCC/MD is constructed. 2D ZCC/MD code denoted by $\left(M \times N, k, \lambda_{a}, \lambda_{c}\right)$ where $M \times N$ represents code size of $2 \mathrm{D} Z \mathrm{ZC} / \mathrm{MD}, k$ is code weight, $\lambda_{a}, \lambda_{c}$ represent auto and cross correlation respectively. Let $Y\left\{y_{0}, y_{1}, y_{2}, \ldots, y_{N-1}\right\}$ and $X\left\{x_{0}, x_{1}, x_{2}, \ldots, x_{M-1}\right\}$ represent 1D-MD code and 1D ZCC code sequences respectively. Some examples for 2d ZCC/MD sequences are shown in Table 1 for $k_{1}=3, k_{2}=2$. 2D hybrid ZCC/MD codes can be created by $g=\{1,2,3, \ldots, M\}$ and $h=\{1,2,3, \ldots, N\} . X_{h}$ represents the patterns for space, $Y_{g}$ represents spreading patterns of spectral.

$$
Z C C=\left[\begin{array}{l}
101000100000 \\
010010001000 \\
000000010101
\end{array}\right] M D=\left[\begin{array}{l}
100001 \\
010010 \\
001100
\end{array}\right]
$$

Table 1. Example of 2-D ZCC/MD Code Sequences for $k_{1}=3$ and $k_{2}=2$

\begin{tabular}{|c|c|c|c|}
\hline$A_{g, h}$ & [101000100000] & {$[010010001000]$} & {$[000000010101]$} \\
\hline$[1]$ & {$[101000100000]$} & {$[010010001000]$} & {$[000000010101]$} \\
\hline 0 & 000000000000 & 000000000000 & 000000000000 \\
\hline 0 & 000000000000 & 000000000000 & 000000000000 \\
\hline 0 & 000000000000 & 000000000000 & 000000000000 \\
\hline 0 & 000000000000 & 000000000000 & 000000000000 \\
\hline$L_{1}$ & ] & {[} & L \\
\hline
\end{tabular}

The derivation of cross-correlation of the 2D ZCC/MD code is based on four matrices of features $A(d), d \in$ $(0,1,2,3)$ is introduced by KERAF, et al. (2016)[10]. Following similar assumption, the cross correlation feature as $A(d)$ can be depicted as follows:

$$
\begin{aligned}
& A^{0}=Y^{T} X \\
& A^{1}=Y^{T} \bar{X} \\
& A^{2}=\overline{Y^{T}} X \\
& A^{3}=\overline{Y^{T} X}
\end{aligned}
$$

$\bar{X}, \bar{Y}$ parameters represent $X \&$ complementary respectively. The 2D hybrid ZCC/MD code cross correlation $A^{(d)}$ and $A_{g, h}$ is shown as follow:

$$
R^{(d)}(g, h)=\sum_{M=1}^{M-1} \sum_{N=1}^{N-1} a_{i j}^{(d)} a_{(i+g)(j+h)}
$$

Wherever $a_{i j}^{(d)}$ represents the $(i, j)_{t h}$ of $A^{(d)}$ and $a_{(i+g)(j+h)}$ is the $(i, j)_{t h}$ of $A_{g, h}$. 2D ZCC/MD code cross correlation given by the (5) can be described on the Table 2.

Table 2. Cross-Correlation of 2D Hybrid ZCC/MD Code

\begin{tabular}{ccccc}
\hline$A_{g, h}$ & $R^{(0)}$ & $R^{(1)}$ & $R^{(2)}$ & $R^{(3)}$ \\
\hline$g=0, h=0$ & $k_{1} k_{2}$ & 0 & 0 & 0 \\
$g=0, h \neq 0$ & 0 & $k_{1} k_{2}$ & 0 & 0 \\
$g \neq 0, h=0$ & 0 & 0 & $k_{1} k_{2}$ & 0 \\
$g \neq 0, h \neq 0$ & 0 & 0 & 0 & $k_{1} k_{2}$ \\
\hline
\end{tabular}

The cross correlation of $A_{0,0}^{0}$ and $A_{g, h}$ can be written:

$$
\begin{aligned}
R^{(0)}(g, h) & =\sum_{i=1}^{M-1} \sum_{j=1}^{N-1} a_{i, j}^{(0)} a_{i, j}(g, h) \\
& =\left\{\begin{array}{lr}
k_{1} k_{2} & \text { for } g=0, h=0 \\
0 & \text { otherwise }
\end{array}\right.
\end{aligned}
$$


According to the Gaussian approximation, BER is calculated. The photodiode is used to obtain the photocurrent and to detect thermal lights as in $[15,16]$ :

$$
\begin{aligned}
\left\langle i_{\text {noise }}^{2}\right\rangle & =\left\langle i_{\text {PIIN }}^{2}\right\rangle+\left\langle i_{\text {shot }}^{2}\right\rangle+\left\langle i_{\text {thermal }}^{2}\right\rangle \\
& =B I^{2} \tau+2 e B I+\frac{4 K_{b} T_{n} B}{R_{L}}
\end{aligned}
$$

$e$ represents electron's charge, $K_{b}$ is constant of Boltzmann, $I$ stands for the average photocurrent, $R_{L}$ is the load resistance, $T_{n}$ is the unlimited temperature, $B$ means the electrical bandwidth. The 2D ZCC/MD code system represents a property of zero cross correlation, so the impact of Multiple Access Interference has been removed. Add on, adapting the same process as [12] where the probability of distributing bit 1 for each user is equal. At the receiver $(0,0)$, the output currents is count on the cross-correlation of $A_{0,0}^{(0)}$ and $A_{g, h}$ as:

$$
\begin{aligned}
I & =R \int_{0}^{\infty} G(v) d v \\
& =\int_{0}^{\infty} \frac{P_{s r}}{k_{2} \Delta v} \sum_{1}^{w} d(w) R^{0}(i, j) U(v, i) d v \\
& =\frac{P_{s r} \Delta v}{k_{2} \Delta v M}\left[k_{1} k_{2}+\sum_{1}^{w} d(w) R^{0}(i, j)\right] \\
& =\frac{R P_{s r} k_{1}}{M}
\end{aligned}
$$

The expression of equations for Shot noise, PIIN and Thermal noise could be expressed as below:

$$
\begin{aligned}
& \left\langle i_{\text {shot }}^{2}\right\rangle=2 e B \frac{R P_{s r} w_{2}}{W} \\
& \left\langle i_{P I I N}^{2}\right\rangle=B \frac{R^{2} P_{s r}^{2} w_{2} k_{1}}{\Delta v W} \\
& \left\langle i_{\text {thermal }}^{2}\right\rangle=\frac{4 K_{b} T_{n} B}{R_{L}}
\end{aligned}
$$

So:

$$
\left\langle i_{\text {noise }}^{2}\right\rangle=2 e B \frac{R P_{s r} w_{2}}{W}+\frac{R^{2} P_{s r}^{2} w_{2} k_{1}}{\Delta v W}+\frac{4 K_{b} T_{n} B}{R_{L}}
$$

Where:

$P_{s r}$ : effective source power at the receiver,

$R:$ The responsivity,

$k_{1}$ : The code weight for spectral sequences,

$k_{2}$ : The code weight for spec spatial sequences,

$W$ : The number of simultaneous clients,

$N$ : lengths of spatial code sequences,

$M$ : length of spectral code sequences.

Remark: at a different time, the probability of sending bit " 1 " for every user at a different time is being $1 / 2$ so:

$$
\left\langle i_{\text {noise }}^{2}\right\rangle=\frac{e B R P_{s r} w_{2}}{W}+\frac{R^{2} P_{s r}^{2} w_{2} k_{1}}{2 \Delta v W}+\frac{4 K_{b} T_{n} B}{R_{L}}
$$

The SNR at the receiver can be written as follow:

$$
\mathrm{SNR}=\frac{\mathrm{I}^{2}}{\left\langle\mathrm{i}_{\text {noise }}^{2}\right\rangle}=\frac{\left[\frac{\mathrm{RP}_{\mathrm{sr}} \mathrm{w}_{2}}{\mathrm{~W}}\right]^{2}}{\frac{\mathrm{R}^{2} \mathrm{P}_{\mathrm{sr}}^{2} \mathrm{w}_{2} \mathrm{k}_{1}}{2 \Delta \mathrm{vW}}+\frac{\mathrm{eBRP}_{\mathrm{sr}} \mathrm{w}_{2}}{\mathrm{~W}}+\frac{4 \mathrm{~K}_{\mathrm{b}} \mathrm{Tn}_{\mathrm{n}} \mathrm{B}}{\mathrm{R}_{\mathrm{L}}}}
$$

From SNR the BER can be derived as depicted [17]: 


$$
B E R=\frac{\operatorname{erfc}(\sqrt{S N R / 8})}{2}
$$

Wherever

$$
\operatorname{erfc}=\frac{2}{\sqrt{\pi}} \int_{0}^{\infty} \exp \left(-y^{2}\right) d y
$$

The listed parameters that applied for analysing the effect of 2D ZCC/MD on the OCDMA performance is shown in Table 3. The following figures depict the analytical results.

Table 3. The Parameters Employed in Numerical Calculations

\begin{tabular}{cc}
\hline Spectral width of broadband & $\Delta \lambda=300 \mathrm{~nm}(\Delta \lambda=3.75 \mathrm{THZ})$ \\
light source & $R=0.75$ \\
PD quantum efficiency & $\lambda_{\mathbf{0}}=1.55 \mu \mathrm{m}$ \\
Operating wavelength & $R_{b}=622 \mathrm{Mbps}$ \\
Data transmission rate & $B=320 \mathrm{MHz}$ \\
Electrical bandwidth & $R_{L}=1030 \Omega$ \\
Receiver load resistor & $T_{n}=300 \mathrm{~K}$ \\
Receiver noise temperature & $e=1.60217646 \times 10^{-19}$ coulombs \\
Electron's charge & $K_{b}=1.38 \times 10^{-23} \mathrm{~W} / \mathrm{K} / \mathrm{Hz}$ \\
Constant of Boltzmann
\end{tabular}

\section{RESULTS AND DISCUSSIONS}

Figure 1 represents BER versus number of simultaneous user when the received effective power Psr $=10^{-2}$ watt and data rate $622 \mathrm{Mbps}$ for proposed code $2 \mathrm{D} \mathrm{ZCC} / \mathrm{MD}(\mathrm{M}=23, \mathrm{~N}=7)$ comparing with 2D DPDC (M=91, N=3) and 2D FCC/MDW (M=23, N=7). It is clear that 2D ZCC/MD can accommodate 360 active user at the BER equal to $10^{-9}$ where the other code accommodate less than 100 user. 2D ZCC/MD represents a better performance compared with other codes.

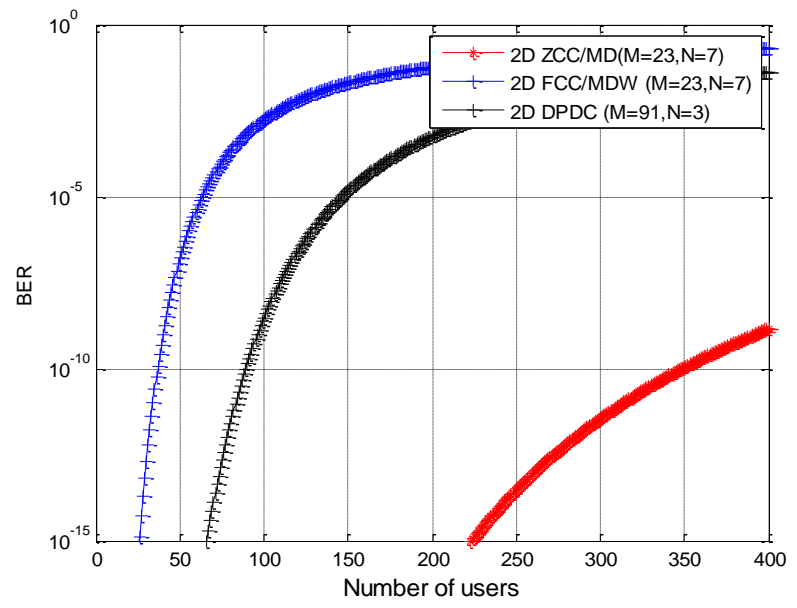

Figure 1. BER versus number of users when $P s r=10^{-2}$ watt and data rate $R b=2.5 G H Z$ comparison with 2D codes FCC/MDW and 2D DPDC

Figure 2 represents the plot of BER versus effective power $P_{s r}$ of the new code 2D ZCC/MD $(\mathrm{M}=23, \mathrm{~N}=7)$ comparing with 2D DPDC $(\mathrm{M}=91, \mathrm{~N}=3)$ and 2D FCC/MDW $(\mathrm{M}=23, \mathrm{~N}=7)$ when number of user equal to 150 and data rate $R b=622 \mathrm{Mbps}$ for every client. It is obvious that when $P_{s r}$ is less than $-25 \mathrm{dBm}$, all codes exhibited poor performance where their BER are higher than $10^{-9}$ domination of noise power represented by thermal noise on the signal power. When the received power increases above $-25 \mathrm{dBm}$, the performance of these codes gradually improve with the power. 2D ZCC/MD exhibit superior performance compared with other codes, where the BER value at $-10 \mathrm{dBm}$ is $10^{-25}$ compared to $10^{-6}$ 
For 2D FCC/MDW and $10^{-6}$ For 2D DPDC. The figure proves that whenever the received power increases, the behavior of $2 \mathrm{D} \mathrm{ZCC} / \mathrm{MD}$ is better than others.

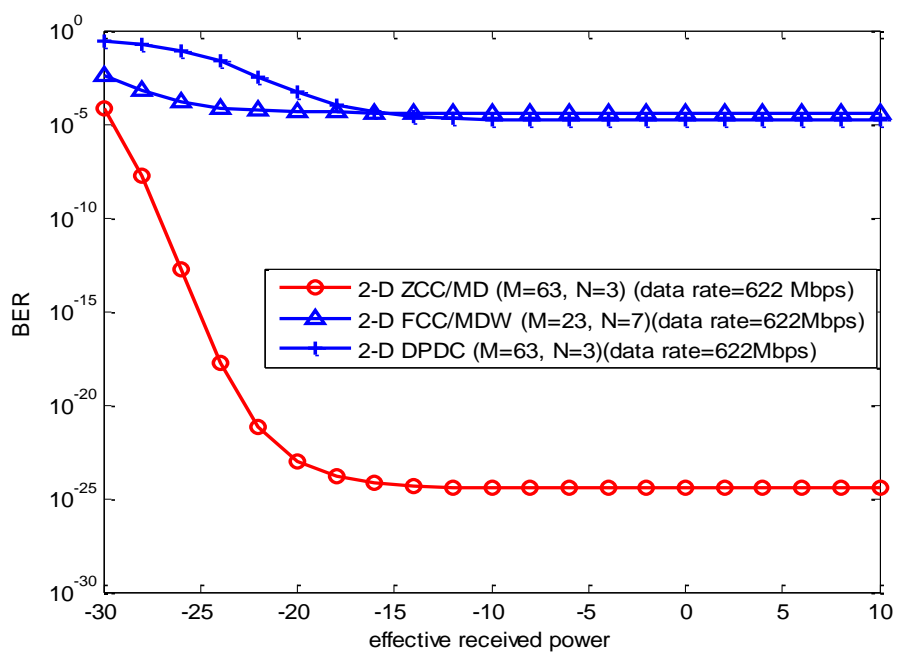

Figure 2. BER versus effective power when data rate $R b=622 \mathrm{Mbps}$ and number of user $K=150$

Figure 3 represents BER versus effective power for 2D ZCC/MD with different data bit rate when it's equal to $622 \mathrm{Mbps}, 1.25 \mathrm{Gbps}$ and $2.5 \mathrm{Gbps}$. At BER equal to $10^{-9} 2 \mathrm{D} \mathrm{ZCC} / \mathrm{MD}$ require $-25 \mathrm{dBm}$ for $R b=622 \mathrm{Mbps}$ while for $R b=1.25 \mathrm{Gbps}$ the effective power equal to $-22 \mathrm{dBm}$. it can be clear that when data rate is equal to $622 \mathrm{Mbps}$ represent a better performance comparing with other value of data rate.

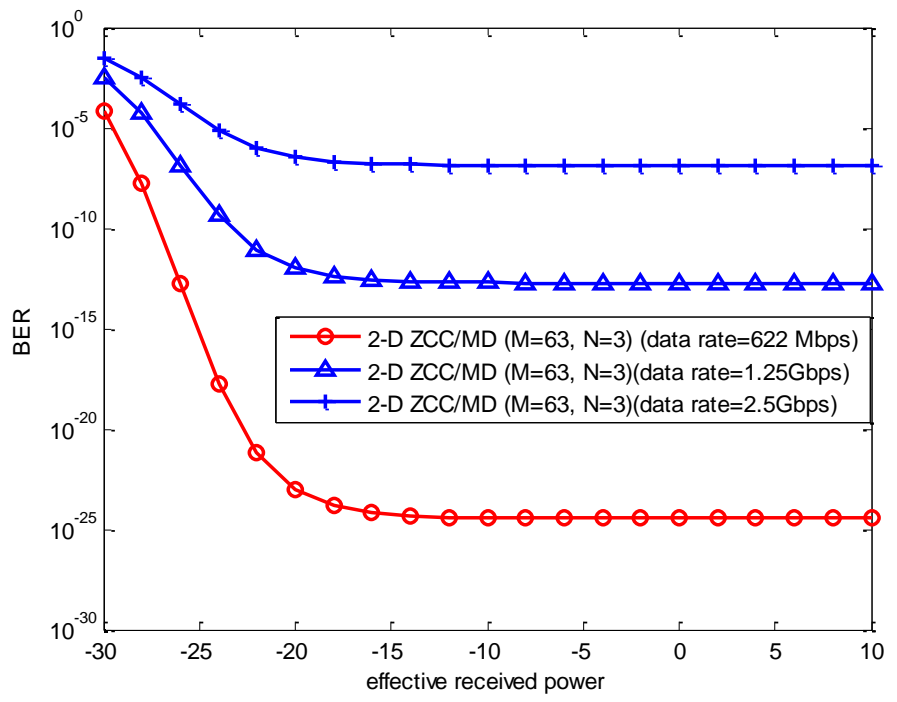

Figure 3. BER versus effective power when data rate equal to $622 \mathrm{Mbps}, 1.25 \mathrm{Gbps}$ and $2.5 \mathrm{Gbps}$ and number of user $\mathrm{K}=150$ for $2 \mathrm{D} \mathrm{ZCC} / \mathrm{MD}$

\section{CONCLUSION}

Through this study a new code for two-dimensional hybrid spectral/spatial OCDMA code named 2D ZCC/MD has been proposed. The impact of PIIN noise, shot noise and thermal noise has been considered. Based on the numerical results as well as the comparing with other codes, the proposed code has a good performance and it can expand more active user with low bit error rate. 


\section{ACKNOWLEDGEMENTS}

The author would like to acknowledge the support from Fundamental Research Grant Scheme (FRGS) under Prof. Dr. Syed Alwee Aljunid, Department of Higher Education, Ministry of Education Malaysia for the providing the grant.

\section{REFERENCES}

[1] Kakaee, M. H., Seyedzadeh, S., Fadhil, H. A., Anas, S. B. A., \& Mokhtar, M. (2014). Development of multiservice (MS) for SAC-OCDMA systems. Optics \& Laser Technology, 60, 49-55.

[2] Aljunid, S. A., Ismail, M., Ramli, A. R., Ali, B. M., \& Abdullah, M. K. (2004). A new family of optical code sequences for spectral-amplitude-coding optical CDMA systems. IEEE photonics technology letters, 16(10), 2383-2385.

[3] Kumawat, S., \& Kumar, M. R. (2016). Generalized optical code construction for enhanced and Modified Double Weight like codes without mapping for SAC-OCDMA systems. Optical Fiber Technology, 30, 72-80.

[4] Rashidi, C. B. M., Aljunid, S. A., Ghani, F., Fadhil, H. A., \& Anuar, M. S. (2012). Cardinality Enhancement Using Flexible Cross Correlation (FCC) Code for Spectral Amplitude Coding Optical Code Division Multiple Access Systems. Journal of Applied Sciences Research, 8(12), 5614-5626.

[5] Mostafa, S., Mohamed, A. E. N. A., El-Samie, F. E. A., \& Rashed, A. N. Z. (2015). Performance Analysis of Diagonal Eigenvalue Unity (DEU) Code Using NAND Subtraction and Spectral Direct Detection Techniques and Its Use with Triple-Play-Services in SAC-OCDMA. Wireless Personal Communications,85(4), 1831-1849.

[6] Rashidi, C. B. M., Anuar, M. S., \& Aljunid, S. A. (2010, May). Study of direct detection technique for zero cross correlation code in OCDMA. In Computer and Communication Engineering (ICCCE), 2010 International Conference on (pp. 1-5). IEEE.

[7] Yeh, B. C., Lin, C. H., Yang, C. L., \& Wu, J. (2009). Noncoherent spectral/spatial optical CDMA system using 2-D diluted perfect difference codes. Journal of Lightwave Technology, 27(13), 2420-2432.

[8] Arief, A. R., Aljunid, S. A., Anuar, M. S., Junita, M. N., \& Ahmad, R. B. (2012). Cardinality enhancement of spectral/spatial modified double weight optical code division multi-access system by PIIN suppression. J International for Light and Electron Optics, 24(19).

[9] Aqrab, I. S., Aljunid, S. A., Rashidi, C. B. M., Dulaimi, A., \& Khalil, L. A. (2017). Evaluation of 2-D Wavelength/Time MDW OCDMA Code via Different Detection Techniques.

[10] Keraf, N. D., Aljunid, S. A., Ehkan, P., \& Safar, A. M. (2016). Analysis of Two Dimensional Wavelength/Time FCC-MDW Code in Optical CDMA System. Journal of Theoretical \& Applied Information Technology, 94(1).

[11] Jellali, N., Najjar, M., Ferchichi, M., \& Rezig, H. (2017). Development of new two-dimensional spectral/spatial code based on dynamic cyclic shift code for OCDMA system. Optical Fiber Technology, 36, 26-32.

[12] Kadhim, R. A., Fadhil, H. A., Aljunid, S. A., \& Razalli, M. S. (2014). A new two dimensional spectral/spatial multi-diagonal code for noncoherent optical code division multiple access (OCDMA) systems. Optics Communications, 329, 28-33.

[13] Anuar, M. S., Aljunid, S. A., Saad, N. M., \& Hamzah, S. M. (2009). New design of spectral amplitude coding in OCDMA with zero cross-correlation.Optics Communications, 282(14), 2659-2664.

[14] Anuar, M. S., Aljunid, S. A., Arief, A. R., Junita, M. N., \& Saad, N. M. (2013). PIN versus Avalanche photodiode gain optimization in zero cross correlation optical code division multiple access system. Optik-International Journal for Light and Electron Optics, 124(4), 371-375.

[15] B.C. Yeh, C.H. Lin \& J. Wu. (2010).Noncoherent spectral/spatial OCDMA system using two-dimensional hybrid codes. Journal of Optical Communications and Networking, 2(9), pp. 653-661.

[16] Z. Wei and H. Ghafouri-Shiraz. (2002). "Codes for Spectral Amplitude-Coding Optical CDMA Systems", J. Lightwave Technol., vol. 20, pp. 1284-1289.

[17] E. Inaty, Shalaby, H. M., \& Fortier, P. (2005). On the cutoff rates of a multiclass OFFH-CDMA system. IEEE transactions on communications, 53(2), p.p323-334.

[18] R. Arulmozhiyal and K. Baskaran, "Implementation of a Fuzzy PI Controller for Speed Control of Induction Motors Using FPGA," Journal of Power Electronics, vol. 10, pp. 65-71, 2010.

[19] D. Zhang, et al., "Common Mode Circulating Current Control of Interleaved Three-Phase Two-Level VoltageSource Converters with Discontinuous Space-Vector Modulation," 2009 IEEE Energy Conversion Congress and Exposition, Vols 1-6, pp. 3906-3912, 2009.

[20] Z. Yinhai, et al., "A Novel SVPWM Modulation Scheme," in Applied Power Electronics Conference and Exposition, 2009. APEC 2009. Twenty-Fourth Annual IEEE, 2009, pp. 128-131. 